\title{
Metformin to Augment Strength Training Effective Response in Seniors (MASTERS): study protocol for a randomized controlled trial
}

Doug E. Long', Bailey D. Peck', Jenny L. Martz², S. Craig Tuggle², Heather M. Bush³, Gerald McGwin², Philip A. Kern ${ }^{4}$, Marcas M. Bamman ${ }^{2}$ and Charlotte A. Peterson ${ }^{1 *}$

\begin{abstract}
Background: Muscle mass and strength are strong determinants of a person's quality of life and functional independence with advancing age. While resistance training is the most effective intervention to combat age-associated muscle atrophy (sarcopenia), the ability of older adults to increase muscle mass and strength in response to training is blunted and highly variable. Thus, finding novel ways to complement resistance training to improve muscle response and ultimately quality of life among older individuals is critical. The purpose of this study is to determine whether a commonly prescribed medication called metformin can be repurposed to improve the response to resistance exercise training by altering the muscle tissue inflammatory environment.

Methods/design: Individuals aged 65 and older are participating in a two-site, randomized, double-blind, placebocontrolled trial testing the effects of metformin or placebo on muscle size, strength, and physical function when combined with a progressive resistance training program. Participants consume $1700 \mathrm{mg}$ of metformin per day or placebo for 2 weeks before engaging in a 14-week progressive resistance training regimen, with continued metformin or placebo. Participants are then monitored post-training to determine if the group taking metformin derived greater overall benefit from training in terms of muscle mass and strength gains than those on placebo. Muscle biopsies are taken from the vastus lateralis at three time points to assess individual cellular and molecular adaptations to resistance training and also changes in response to metformin.

Discussion: The response of aged muscles to a resistance training program does not always result in a positive outcome; some individuals even experience a loss in muscle mass following resistance training. Thus, adjuvant therapies, including pharmacological ones, are required to optimize response to training in those who do not respond and may be at increased risk of frailty. This is the first known metformin repurposing trial in non-diseased individuals, aimed specifically at the resistance exercise "non-responder" phenotype present in the aging population. The overall goal of this trial is to determine if combined exercise-metformin intervention therapy will benefit older individuals by promoting muscle hypertrophy and strength gains, thereby maintaining functional independence.
\end{abstract}

Trial registration: ClinicalTrials.gov, NCT02308228. Registered on 25 November 2014.

Keywords: Metformin, Placebo, Sarcopenia, Aging, Skeletal muscle, Resistance exercise, Inflammation, Medication

\footnotetext{
*Correspondence: cpete4@uky.edu

Philip A. Kern, Marcas M. Bamman and Charlotte A. Peterson Multi-Principal

Investigators

${ }^{1}$ College of Health Sciences and Center for Muscle Biology, University of

Kentucky, Lexington, KY, USA

Full list of author information is available at the end of the article
} 


\section{Background}

Muscle mass and strength are critical determinants not only of a person's quality of life and functional independence, but also metabolic health, as skeletal muscle is the primary regulator of glucose uptake, usage, and storage. The aged suffer obligatory losses of muscle mass and strength, exacerbated by illness and physical inactivity, and in the absence of effective countermeasures, advancing age leads to physical frailty and dependent living. The significance of this cannot be overstated, as the consequent impact on individual life quality and systemwide healthcare expenditures is staggering, and compounded by the ever-expanding elderly population. Muscle mass and strength decrease approximately $10 \%$ per decade after the age of 50 , with strength loss being even more pronounced after the age of 70 [1]. While resistance exercise training is an established method to increase muscle mass, strength, and functional capacities such as balance and mobility, this effect can be very low in aging cohorts $[2,3]$. In fact, up to $38 \%$ of older adults do not respond with significant muscle growth when performing resistance exercises alone [4]. Thus, identifying strategies to improve muscle health and applying therapies that may be combined with resistance exercise to slow the debilitating conditions associated with the aging process are of paramount importance. This protocol was designed to study the molecular and cellular mechanisms underlying the "non-responder" phenotype with the goal of identifying a novel intervention to enhance muscle adaptation to progressive resistance exercise training (PRT) in healthy, elderly individuals. A randomized controlled trial is currently underway to study whether metformin, a first-line drug of treatment for type 2 diabetes, can be used alternatively as a treatment to improve the ability of older individuals to respond appropriately to PRT (Fig. 1).

Recent work has shown that advanced age promotes an inflammatory muscle microenvironment which may contribute to the exercise "non-responder" phenotype [5-8]. Pro-inflammatory cytokines such as interleukin-6 (IL-6) and tumor necrosis factor alpha (TNF- $\alpha$ ) have elevated expression levels both pre- and post-resistance training in seniors compared to their younger counterparts, with TNF- $\alpha$ being a key prognosticator for non-responders following a 16-week resistance training intervention [6, 9]. Higher levels of these inflammatory cytokines have been associated with lower muscle mass and strength [9]. Additionally, it has been suggested that aging results in substandard muscle macrophage function, hindering the regulation of inflammation and ultimately the regenerative capacity of skeletal muscle [10].

Muscle macrophages have been most studied within the context of muscle damage and regeneration in rodents, where they have been shown to progress from a phagocytic, inflammatory M1 state to an antiinflammatory M2 state that promotes repair [11-14]. M2 macrophages have also been reported to protect against muscle atrophy in rodents and promote muscle recovery both in vivo and in vitro [15]. Many of the factors produced by M2 macrophages facilitate muscle growth and repair by stimulating the activity of muscle stem cells called satellite cells $[16,17]$. The resolution of inflammation following injury in muscle is a product of both repressed inflammatory cytokine production and transcriptional up-regulation of anti-inflammatory genes like insulin-like growth factor (IGF)-1 and IL-4 and IL10 from M2 macrophages. We showed that resistance exercise in humans that promotes muscle growth results in an increase in the relative proportion of alternatively activated M2 macrophages, with only a small subpopulation expressing classical, pro-inflammatory M1 characteristics 3 days after the exercise bout, and this response was impaired in the elderly [10]. Thus, evidence supports the idea that augmenting M2 macrophage abundance in muscle will facilitate a hypertrophic response.

The use of pharmacological treatments that moderate the inflammatory microenvironment of aged muscle is of particular importance to the success of exercise interventions such as PRT. Metformin has come to the forefront due to recent negotiations undertaken by experts in the aging field with the Food and Drug Administration (FDA) for the classification of an anti-aging drug, whose widespread prescription could benefit our growing aged population [18]. Metformin is a biguanide compound, prescribed since the early 1980s as an antihyperglycemic agent for prediabetes and type 2 diabetes. Re-evaluation and potential re-classification stem from longitudinal studies carried out in diabetic patients subject to $10+$ years of metformin treatment [19]. The diabetic subjects receiving metformin monotherapy demonstrated a $15 \%$ higher survival rate than the aged matched population over the $10+$ years under observation [19]. This finding supports the position that metformin treatment may be applicable to improving the health span of non-diabetic populations. Metformin primarily exerts its major effects on improving insulin sensitivity through activation of adenosine monophosphateactivated protein kinase (AMPK), a master switch to regulate suppression of hepatic glucose output [20-22]. Recent work shows that skeletal muscle repair may be improved when AMPK is activated [23], and that the AMPK 1 isoform is required for macrophages to acquire the functions of the M2 subtype in muscle [24]. The AMPK $\alpha 1$ isoform in macrophages is the only catalytic subunit expressed, and when in association with an AMPK activator such as metformin (by way of activating transcription factor 3 , ATF-3) or 5-amino-1- $\beta$-D-ribofuranosyl-imidazole4-carboxamide (AICAR), this isoform demonstrates 


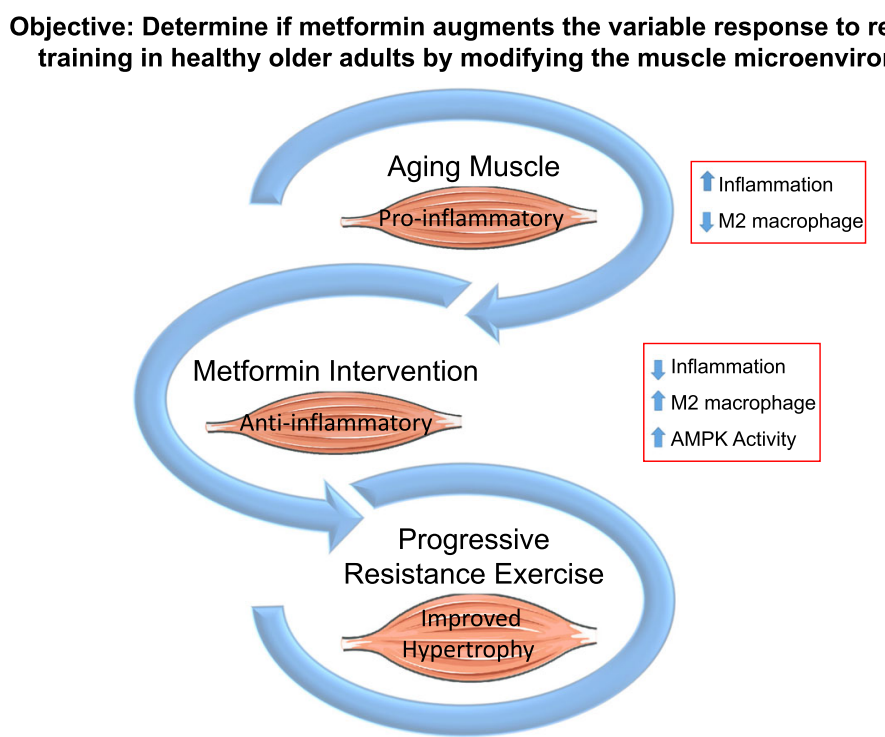

Fig. 1 Does metformin augment the variable response to resistance training in healthy older adults by modifying the muscle microenvironment? Study objective for a randomized control trial of metformin to improve the response of muscle to resistance exercise

the ability to induce M1 to M2 macrophage polarization such as in response to lipopolysaccharide (LPS), an endotoxin with pro-inflammatory effects, over placebo [25]. When AMPK $\alpha 1$ is absent, skeletal muscle repair is delayed, associated with elevated levels of M1 macrophage markers [24].

Interestingly, our preliminary analyses of muscle from insulin resistant participants $(n=6)$ showed that 10 weeks of metformin (1700 mg per day) effectively increased M2 macrophage abundance (Fig. 2a) and decreased inflammatory cytokine gene expression (Fig. $2 \mathrm{~b}$ ) in vastus lateralis muscle biopsies. These provocative findings have led us to our central hypothesis that adjuvant metformin treatment may improve the responses to PRT in the elderly by altering the muscle tissue inflammatory environment, thereby enhancing mechanisms that drive resistance traininginduced myofiber hypertrophy.

\section{Methods/design}

Human subject participation

\section{Trial summary and overall design}

Subjects are participating at two sites, the University of Kentucky (UK) and the University of Alabama at Birmingham (UAB), in a randomized, double-blind, placebo-controlled trial centered on a novel, alternative use of metformin to potentially augment muscle mass gains to resistance exercise. The two-site design ensures that the recruitment goals are met and that the results are generalizable due to the diverse population that could not be studied at either site alone. Complete participation requires that each individual spend approximately 19 weeks attending 8 study assessments and
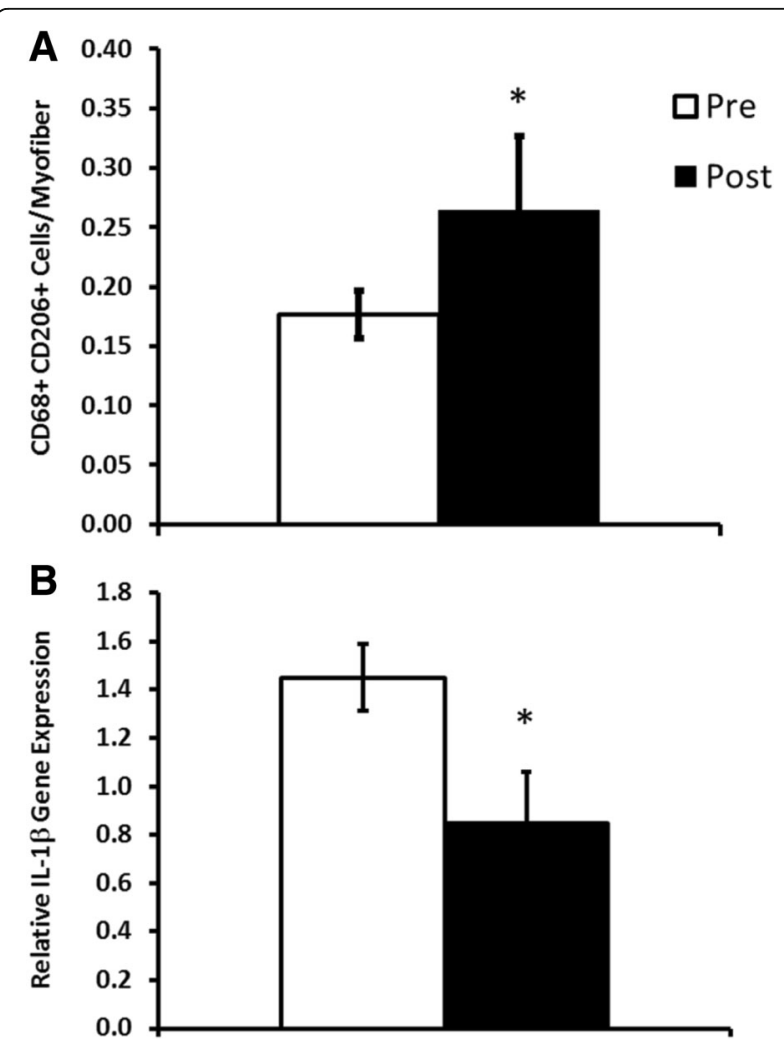

Fig. 2 Preliminary analyses of the effects of metformin on muscle tissue. Effects of metformin on muscle tissue M2 macrophage (CD68 + CD206+) frequency (a) and IL-1 $\beta$ inflammatory gene expression (b). *Different from pre-treatment, $p<0.05$. Values are mean \pm standard error (SE) 
finishing 42 resistance exercise sessions and, thus, approximately 50 total study visits. This time includes a 2-week pre-treatment or baseline period involving a detailed screening and physical exam for inclusion/exclusion clearance as well as baseline testing for glucose tolerance, body composition, and muscle function, a 2-week medication ramp-up period prior to exercise along with medicationonly testing, followed by 14 weeks of supervised PRT. All post-intervention assessments are completed within 3 days following training. In addition, participants can opt to complete two follow-up assessments of muscle function, quality of life, and physical activity levels at approximately 10 and 36 weeks posttraining. The effects of metformin or placebo on the muscle environment after 2 weeks of ramped medication and when combined with 14 weeks of PRT are measured. The protocol is designed in accordance with the Standard Protocol Items: Recommendations for Interventional Trials (SPIRIT) guidelines for interventional trials (Additional file 1). A summary of study visits and the participant data collection schedule are shown in Table 1, and the flow diagram for the overall study in Fig. 3. The study is currently being conducted at each respective Center for Clinical and Translational Science (CCTS) Clinical Research Unit, the Human Performance $\mathrm{Lab}$ at UK, and the Center for Exercise Medicine at UAB where subjects are compensated $\$ 300$ for their time. Subjects are stratified and randomized based on study site and functional status.

\section{Recruitment and enrollment}

One hundred and twenty men and women $\geq 65$ years of age ( $n=72$ at UAB, $n=48$ at UK), representative of the racial and ethnic makeup of greater Birmingham, Alabama and greater Lexington, Kentucky, are being recruited through general advertisements and volunteer databases. Given the anticipated attrition rate of $20 \%$, this will allow us to achieve a final sample size of 100 . Ethnic and racial groups are expected to enroll based on the proportions of the surrounding areas with emphasis placed on targeted recruitment of under-represented minorities. However, representation of these groups may be lower than intended. Individuals will be recruited who are not currently resistance training or participating in other forms of organized exercise more than two times per week, and who have a Short Physical Performance Battery (SPPB) score $>3$ (score range 0-12). These represent non-disabled and mobile individuals who are able to participate in the functional testing and resistance exercise. Recruits are pre-screened by telephone interviews to minimize screen failures due to medical history, and all procedures included in the study are explained in detail (including approximate time commitment and potential risks) to the subjects by a member of the research team designated to do so. Upon an initial visit, subjects undergo a detailed medical history, medication use history, resting electrocardiogram, and physical exam by the study physician or physician assistant. Final enrollment decisions and eligibility are based on inclusion/exclusion criteria, 2-h oral glucose tolerance test (OGTT), and bloodwork also performed during the screening visit. These criteria are shown in Table 2.

\section{Physical function}

Participants complete the SPPB for the assessment of physical function [26]. This battery of tests includes three timed standing balance tests (side-by-side, semitandem, and tandem), a 3-or 4-m habitual gait speed test performed twice, and a timed repeated chair sit to stand (five times). Performance for each set of tasks is scored (0-4), with a summary score of $0-12$. Functional assessment data are collected during pre-treatment, post-training, and during optional follow-up testing and are used as a stratification measure at each site (high vs. low).

\section{Randomization and stratification}

Subjects screening into the study are randomized and stratified by both site (UK vs. UAB) and by SPPB score (4-9 low, vs. 10-12 high) to receive either metformin or placebo. Since recruitment at UAB is expected to be more than at UK, participants will be stratified by site prior to randomization. Randomization is implemented in permuted blocks of 4 using SAS v9.2 PROC PLAN to ensure adequate distribution of all groups across the collection period. Only the statistician and the investigational pharmacy will have access to the randomization and stratification scheme; thus, assignments will be unknown to the investigators and study team. The protocol also appoints an individual outside of study assessments or specimen analysis to reveal group placement to participants at a specified time.

\section{Medication or placebo}

The metformin and placebo tablets were obtained commercially through different facilities. The placebo tablets were obtained from the Veterans Affairs (VA) Cooperative Studies Program Clinical Research Pharmacy Coordinating Center, which is in New Mexico working through the Biomedical Research Institute of New Mexico. This program is registered with the FDA as a manufacturing, packaging, and distribution facility and serves the needs of VA studies, as well as National Institutes of Health (NIH)-funded and some industry studies. There are many different manufacturers of metformin, and the VA facility in New Mexico has provided us with a tablet which is identical to metformin $850 \mathrm{mg}$ tablets that are manufactured by Amneal Pharmaceuticals, 


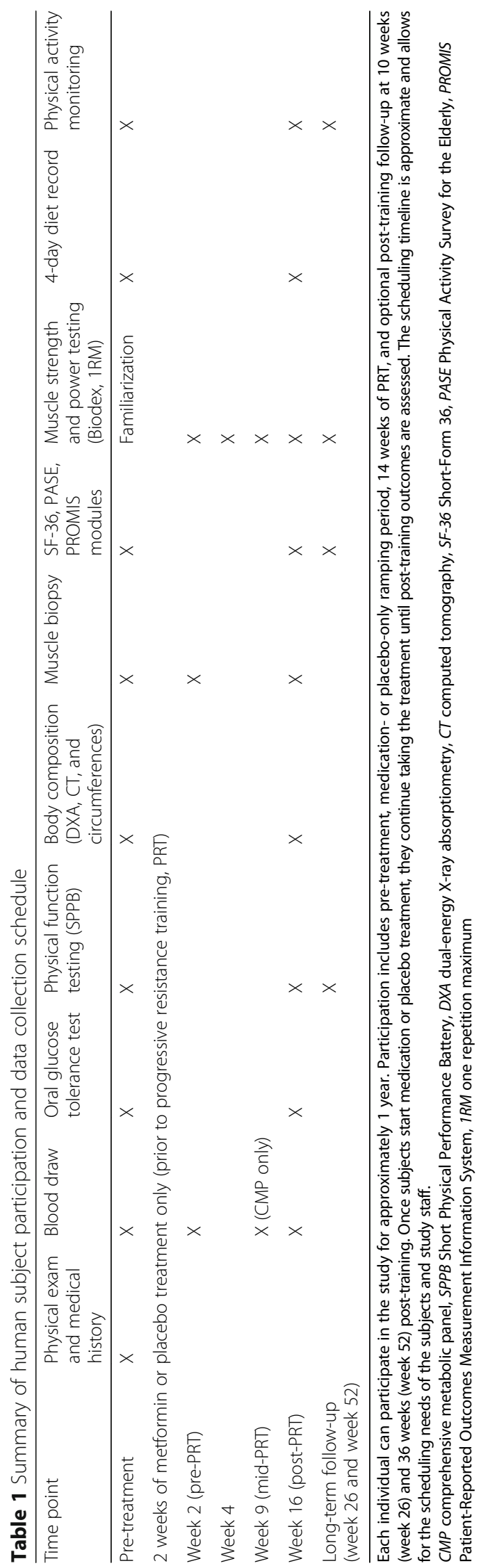




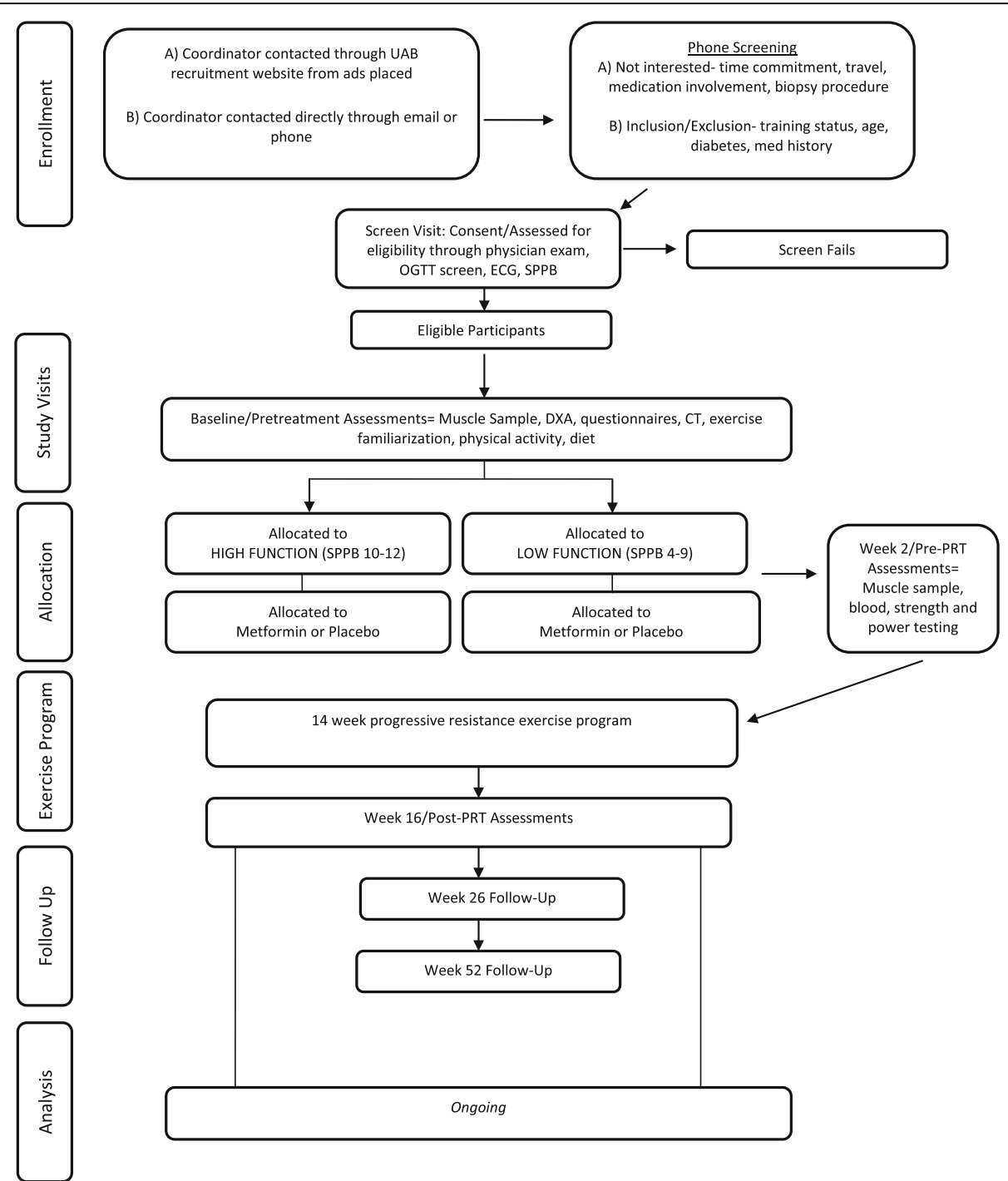

Fig. 3 MASTERS Flow Diagram

an established generic drug maker. Therefore, we purchased generic Amneal $850 \mathrm{mg}$ metformin tablets through our investigational pharmacy. Subjects randomized to metformin will take increasing doses during the medication ramp-up period of 2 weeks to reduce gastrointestinal (GI) side effects as follows: 1 tablet $(850 \mathrm{mg})$ per day for a period of 7 days and 2 tablets per day $(1700 \mathrm{mg})$ for a period of 7 days, the latter being the target clinical dose, continued throughout the 14 weeks of PRT and final week of post-intervention assessments. In some subjects, the dose progression may be slower and may not reach $1700 \mathrm{mg} /$ day due to GI side effects. The study physician makes individualized modifications should the need arise. Subjects remain in the study as long as they can tolerate at least $850 \mathrm{mg} /$ day. These data will be collected and reported. The placebo tablets look identical to the metformin tablets, but contain inert substances, and the escalating dose schedule is the same. The hospital investigational pharmacy dispenses the metformin or placebo in uniform generic bottles at five time points (pretreatment for initial 2-week medication ramp, baseline PRT weeks 2-4, PRT weeks 4-8, PRT weeks $8-12$, and PRT weeks 12-16) throughout the protocol so that compliance can be monitored. This allows for a compliance schedule of approximately 4 weeks where subjects return any unused product. Extra doses are given as needed and are noted by the study coordinator.

\section{Quality of life}

Quality of life is being determined through self-report instruments, the Short Form 36 (SF-36) and the PatientReported Outcomes Measurement Information System (PROMIS), for the effectiveness of the exercise training intervention on different health-related domains including physical, mental, and social well-being. Subjects 
Table 2 Study inclusion and exclusion criteria

Inclusion criteria

$\cdot \geq 65$ years of age

- Independently mobile with an SPPB score 4-12

- Access to transportation

- Capable of providing informed consent (cognitively intact)

- Negative diagnostic (12-lead ECG), submaximal Graded Exercise Testing (GXT) (if applicable)

Exclusion criteria

- Obesity (body mass index (BMI) >30)

- Serum creatinine $>1.4$ because of risk of lactic acidosis with metformin

- History of structured, regular resistance exercise training within the past year (more than two times per week consistently)

- Chronic aspirin or non-steroidal anti-inflammatory drug (NSAID) use (unless it can be safely stopped prior to the biopsies) and any other use of an anticoagulant (e.g., Coumadin) or history of bleeding

- History of alcoholism or liver disease

- History of hypo- or hypercoagulation disorders including subjects taking Coumadin

- Any end-stage disease and/or a life expectancy less than 1 year

- Neurological, musculoskeletal, or other disorder that would preclude them from completing resistance training and all performance tests

- Uncontrolled hypertension, unstable or exercise-induced angina pectoris or myocardial ischemia, or congestive heart failure

- Diabetes mellitus: HgbA1C $>6.5$, or fasting glucose $>126 \mathrm{mg} / \mathrm{dl}$, or 2-h glucose (on oral glucose tolerance test (OGTT)) >200)

- Any other medical condition that would interfere with testing or increase one's risk of complications during exercise, as judged by the study physicians

- Any other condition or events considered exclusionary by the Principal Investigator and/or physician, such as non-compliance

- Lidocaine allergy (1\% lidocaine is the local anesthetic used during the muscle biopsy procedure)

- Currently receiving androgen or anabolic therapy

complete these assessments during pre-treatment, following the resistance exercise program, and during follow-up assessments at 10 and 36 weeks post-training.

\section{Physical activity measures}

The Physical Activity Survey for the Elderly (PASE) as well as physical activity monitors are used in this study. The PASE is a self-report or interview-based measure designed to capture and assess occupational, household, and leisure activities typically performed by older adults including those of lighter intensity. Time spent participating in each activity area is multiplied by a weighted value that reflects the amount of energy expended by an older person engaged in that activity. These weighted values are then summed to yield a composite PASE score which is used for data analysis. This questionnaire has been found to be both reliable and valid among community-dwelling and physically disabled older adults. The physical activity monitor being used in this study is the Fitbit Flex; it is worn on the non-dominant wrist for an assessment of daily step counts over a period of at least 5 days. The day given and day of return are excluded from the analysis, since the subject will not have worn the monitor for a complete day. The measures are taken before resistance exercise training and during the last week of training to account for possible increases in activity due to the exercise sessions. Subjects also complete these measures during their optional follow-up visits by wearing the monitors for a period of 1 week before returning to complete strength and functional measures.

\section{Dietary monitoring}

Participants are asked to maintain their normal dietary intake throughout the study period. Energy intake and macronutrient composition are assessed by 4-day diet records during pre-treatment and during the last week of training. The nutrient content is determined by those qualified to use the Nutrition Data System for Research, which utilizes the multiple-pass method to help improve the validity of dietary data [27].

\section{Progressive resistance exercise training (PRT)}

The 14 weeks of PRT (42 sessions \pm 5 sessions), summarized in Table 3, are supervised by trained personnel such as exercise physiologists or senior-level physical therapy or athletic training graduate students under the supervision of an exercise physiologist. Each site has a certified, lead exercise physiologist with several years of experience, responsible for managing the day-to-day activities and supervising all trainers. Subjects are instructed on proper techniques and continuously monitored. Following a 5-min warm-up on a self-selected bicycle ergometer or treadmill, PRT consists of eight constant load movements to train all major muscle groups. These exercises include those to strengthen the lower body and thigh muscles (leg press, knee extension,

Table 3 Progressive resistance training protocol

\begin{tabular}{llll}
\hline 14 weeks (42 \pm 5 sessions) & & \\
\hline & Monday & Wednesday & Friday \\
\hline Goal & Hypertrophy & Power & Hypertrophy \\
Intensity & $\sim 70 \% 1 \mathrm{RM}$ & $\sim 40 \% 1 \mathrm{RM}$ & $\sim 70 \% 1 \mathrm{RM}$ \\
Reps & $8-12$ & 12 & $8-12$ \\
Sets & 3 & 3 & 3 \\
Rest & $\sim 60 \mathrm{~s}$ & $\sim 30 \mathrm{~s}$ & $\sim 60 \mathrm{~s}$
\end{tabular}

Each session includes eight exercises performed bilaterally in pairs, with indicated rest between pairs (chest press/squat, leg press/calf press, lateral pulldown/leg extension, bicep curl/tricep extension), along with core and trunk exercises. Progression to 3 full sets is achieved by session 5 . Initial exercise intensity is determined by $1 \mathrm{RM}$ for the chest press, leg press, and leg extension, while a 10RM is used for all other exercises. $R M$ repetition maximum 
body weight squat progressing to a split squat if the participant is able, calf press) as well as upper body exercise (chest press, lateral pulldown, biceps curl, and triceps pressdown). Core and trunk exercises are also a part of the routine, including abdominal work (various abdominal exercises) and lower back flexibility and strengthening (alternating supermans). All resistance exercises are performed bilaterally, progressing to reach full volume and intensity for each exercise by the end of the second week. We are implementing a variable-intensity prescription: "high-low-high" 3 days/week program which has previously been shown to optimize muscle mass and strength gains in older adults [28]. On Mondays and Fridays, intensity is high with subjects completing 3 sets of 8-12 repetitions at 10RM with $60-90 \mathrm{~s}$ between sets. Progression is incorporated continuously by incrementing the resistance load when 12 repetitions are completed for 2 of 3 sets. On Wednesdays, resistance loads are reduced $\sim 30 \%$ with 30 - to -60 -s rest periods with the emphasis on more rapid, concentric training (with controlled eccentric loading) to develop explosive power while providing a protracted recovery period between high resistance sessions. Participants complete the routine in supersets combining two exercises, loading antagonistic or uninvolved muscle groups, in succession with minimal rest between exercises and 60- to 90 -s rest periods between supersets. Following resistance exercise, participants complete a 5-min cooldown. Participants aim to complete 42 exercise sessions but have the flexibility to train \pm 5 sessions to account for follow-up testing as well as for participant schedules and vacations, etc. Participants are expected to be in good compliance by completing at least 2 consecutive exercise sessions in a row before follow-up testing. Note that, due to the two-site design, exercise equipment between the sites is different, with UK utilizing pneumatic air-driven Keiser equipment and UAB utilizing standard plate-loaded and weight stack BodyMasters and LifeFitness equipment. Between-site analysis of training outcomes will be performed to account for training effects due to differences in equipment. Details of the resistance training protocol are given in Table 3.

\section{Muscle biopsy}

Subjects have three biopsies during this protocol: at pretreatment, after 2 weeks of metformin or placebo, and at3 days following the last resistance exercise bout. Subjects taking blood thinning medications are asked to stop those medications for a period of 3-5 days prior to each biopsy. Muscle tissue is obtained from the vastus lateralis after administration of local anesthetic (1\% lidocaine premixed with bicarbonate) using a $5 \mathrm{~mm}$ Bergstrom needle with suction. A small incision is made in the skin to allow the needle to be briefly inserted into the muscle so as to obtain approximately $200-300 \mathrm{mg}$ of tissue, usually occurring over two passes. Direct pressure is applied to stop bleeding for approximately $5-7 \mathrm{~min}$, and the wound is closed with Steri-Strips and covered with gauze and a pressure bandage. Biopsies follow a left, right, left leg pattern unless a research subject requests differently. Muscle tissue is divided as follows: $100 \mathrm{mg}$ is processed for muscle cell isolation, $\sim 100 \mathrm{mg}$ is snap-frozen ( $\sim 30 \mathrm{mg}$ aliquots) in liquid nitrogen for RNA and protein isolation, and $\sim 50 \mathrm{mg}$ is mounted in tragacanth gum and frozen in liquid nitrogen-cooled isopentane for detailed immunohistochemical (IHC) analyses. All biopsies are performed with the subjects fasting.

\section{Post-training follow-ups}

The subjects remain in the study after completion of the training program for an additional 36 weeks to be contacted for optional follow-up visits. These visits occur at approximately 10 weeks and 36 weeks following the training period but are optional for the subjects. The retention of strength, function, physical activity, and quality of life is examined. Subjects are not asked to refrain from any activity they would like to participate in including continuing their own strength training. Metformin or placebo is discontinued during this time. Changes in muscle strength and power are plotted against all time points shown in Table 1 . We assess strength change from the leg extension exercise due to its isolation of the vastus lateralis and the leg press as a secondary measure. Muscle power is assessed from the isotonic mode on the Biodex set at $40 \%$ of each subject's maximum voluntary isometric contraction. Insulin sensitivity is measured from plasma glucose and insulin values during a 2-h OGTT. The Homeostatic Model Assessment of Insulin Resistance (HOMA-IR) is calculated from fasting blood samples while the Matsuda index is utilized for all other samples.

\section{Data safety monitoring}

The current clinical trial has obtained the required institutional review board (IRB) approval. Both sites have conducted similar trials in the past and are experienced with all regulatory requirements and with working with their IRBs. The study has eligibility requirements to ensure that subjects enrolled have an appropriate riskto-benefit ratio. The Principal Investigators (PIs) are ultimately responsible for keeping all study documents updated and available for inspection by the sponsor, the UAB and UK IRBs, and other authorized reviewers. Both sites report in the same manner and in the same time frame. Monitoring for adverse events (AEs) is conducted in real time by the study investigators and study coordinators. Risks involved with this study are considered 
greater than minimal risk and are listed in the consent form. For this reason, we have utilized the standing independent Data Safety Monitoring Board (DSMB) as chartered by the UK CCTS to monitor the safety of this study at both sites. The DSMB reviews protocol performance, regulatory requirements, particularly the reporting of AEs, and serves as the sole DSMB for the study. Both sites use the same standardized AE report for the DSMB review to allow for an effective assessment of potential issues. The DSMB review is given to the PIs and study coordinators, who can then report to the UK and UAB IRBs during Continuation Reviews.

The study coordinator is in constant contact with subjects to assess pain, infection, and other symptoms indicating possible post-procedure AEs. Subjects are discharged from the Clinical Research Unit with specific selfmonitoring guidelines and instructed to call immediately regarding any concerning signs or symptoms. The study procedures are halted at any time a serious safety concern is noted.

AEs are graded according to intensity and relatability to the study. Annual reporting of AEs and serious adverse events are conducted with the IRB Continuation Review in their appropriate time frames according to their protocol.

\section{Outcome measures and analyses \\ Primary outcomes}

The primary outcomes are as follows:

\section{Muscle size}

a) Myofiber cross-sectional area (CSA)

b) Computed tomography $(\mathrm{CT})$ skeletal muscle area of the right thigh

c) CT skeletal muscle area of the vastus lateralis

2. Muscle strength and power
a) $1 \mathrm{RM}$ on the leg extension exercise
b) Biodex maximum voluntary isometric contraction (MVIC)
c) Biodex isotonic power at $40 \% \mathrm{MVIC}$

Muscle size This involves individual fiber CSA and total thigh muscle size/mass. The CSA at the myofiber level from vastus lateralis muscle biopsies is the primary outcome of interest. Fiber type-specific CSA is quantified on 7- $\mu \mathrm{m}$ serial cryosections using an antibody- recognizing laminin to delineate individual myofibers, followed by incubation with a battery of monoclonal antibodies against the different myosin heavy chain $(\mathrm{MyHC})$ isoforms. The three MyHC primary antibodies (types I, IIa, and IIx) are of different isotypes so that all primary antibodies are added to the sections simultaneously followed by isotype-specific secondary antibodies conjugated to different fluorescent tags. Digital images are captured of the entire cross section (between 400-1200 fibers), and mean myofiber CSA by fiber type quantified using a recently developed automated algorithm.

Muscle size is determined using a single slice CT image collected on a GE Discovery CT750 HD at UAB and Siemens Somatom Definition at UK at the midthigh defined as the midpoint between the inguinal crease and the proximal border of the patella with the hip and knee flexed $\sim 90^{\circ}$. CT images are used to quantify skeletal muscle and fat area of the right thigh of each subject using $100 \mathrm{~mA}$ with a scanning time of $3 \mathrm{~s}$ and a $512 \times 512$ matrix. With the subject supine, one $5-\mathrm{mm}$ thick cross section scan of the leg is taken by lining the scan to the midpoint mark identified on each participant. The feet of each participant are wrapped to minimize movement. Tissue area quantification is determined using corresponding attenuation values of $\geq 200 \mathrm{HU} ;-190$ to -30 $\mathrm{HU}$; and $0-100 \mathrm{HU}$ for bone, adipose tissue, and skeletal muscle, respectively, using available software (NIH Image); http://rsbweb.nih.gov/ij/). Skeletal muscle is subdivided into areas of low attenuation (0-34 HU) representing fat-rich muscle, and high attenuation values $(35-100 \mathrm{HU})$ representing muscle with normal fat content. Each subject receives a $\mathrm{CT}$ scan during pre-treatment and 1-3 days following the last resistance training session. The total skeletal muscle areas of the right mid-thigh, quadriceps muscle area, and isolated vastus lateralis are quantified.

Muscle strength and power Voluntary, dynamic strength is evaluated by testing a one repetition max (1RM), defined as the maximal load that a subject can lift one time with proper form through a full range of motion, via our well-established methods that have been standardized across sites $[8,29,30]$. After 2 sets of warm-ups at an estimated $40-50 \%$ for the first set and $70-80 \%$ for the second set, single repetition trials, separated by 1-2 min of rest, are performed with increasing resistance until two failed attempts at a given load. The last successfully lifted load with good form and range of motion will be recorded as the 1RM. Verbal encouragement is given during all lifts, and $1 \mathrm{RM}$ is always completed during high or heavy days as described above.

We also evaluate MVIC knee extension strength with a knee angle of $60^{\circ}$ using our established methods on a Biodex 4 dynamometer, available at both sites [31]. One set of 5 reps is also being completed by the subjects to determine knee extension concentric power using the Biodex set on isotonic mode to allow for variable velocities with a constant external load equal to $40 \%$ of maximum voluntary isometric strength. Peak power is recorded as the highest power recorded during the 5 repetitions. Subjects complete these assessments 5 times throughout the protocol including a familiarization session during pre-treatment, after 2 weeks of metformin 
or placebo, after 2 weeks of resistance training (week 4), at the midpoint of the resistance training program (week 9), and during their last week of training (week 16). Familiarization sessions are designed to accustom the subjects to the different exercises using proper technique and explain 1RM testing (maximum efforts were not given on 1RMs). However, maximum efforts are given on the Biodex to be used for comparison with the effort given during the 2-week metformin or placebo testing period. Week 4, after 2 weeks of resistance training, is used as the baseline 1RM strength to account for the initial neuromuscular adaptations occurring at the beginning of the resistance training program.

\section{Secondary outcomes}

Secondary outcomes include the following:

1. Body composition: dual-energy X-ray absorptiometry (DXA) total and thigh mineral-free lean mass, waist, abdominal, and hip circumferences

2. Insulin sensitivity

a) Fasting plasma glucose and insulin (HOMA)

b) Matsuda index based on glucose and insulin values

3. Physical activity and quality-of-life self-reports

Thigh muscle mass Regional thigh DXA mineral-free lean mass is used as a secondary indicator of muscle response. DXA scans are performed for body composition (whole body, regional fat, and lean mass) and bone mineral density assessments using a Lunar Prodigy (UAB) and an IDXA (UK) using standardized methods for regional partitioning. Data quality is assured by phantom calibrations, and each participant receives a DXA scan at two time points during the study, pre-treatment and post-resistance training, by study personnel trained in this procedure. The subjects are instructed to remove all objects such as jewelry or eyeglasses and to wear a hospital gown, or a lightweight shirt and shorts containing no metal during the scanning procedure. All scans are analyzed by a trained and certified investigator using the GE Lunar software v10.0. DXA bone mineral content (BMC; $\mathrm{kg}$ ), DXA bone mineral density (BMD; $\left.\mathrm{g} / \mathrm{cm}^{2}\right)$, DXA fat-free mass (FFM; $\mathrm{kg}$ ), DXA mineral-free lean mass (MFL; kg), DXA fat mass (Fat; kg), and DXA percent fat (\%Fat) are assessed. Furthermore, custom analyses are performed to determine femur length and right and left thigh muscle and fat mass. Femur length is measured from the center of the junction of the femoral head (at the femoral neck) and the acetabulum to the center of the bottom of the medial condyle. Right and left thigh muscle and fat mass are calculated by subtracting the lower leg from the respective right or left leg total mass by creating a custom region of interest (ROI) through the center of the knee joint between the tibial plateau and the femoral condyles and encapsulating the lower leg past the toes. In addition to DXA, circumferences are taken at three sites using American College of Sports Medicine (ACSM) standardized procedures including the waist defined as the narrowest part of the torso, the abdomen at the level of the umbilicus, and the hips defined as the maximal circumference of the buttocks.

Fasting blood glucose and insulin and oral glucose tolerance test (OGTT) Subjects undergo four blood draws and complete two OGTTs in order to assess fasting glucose and insulin, and also to assess safety and eligibility requirements (i.e., creatinine, glucose, liver enzymes, lipids, thyroid-stimulating hormone (TSH), complete blood count ( $\mathrm{CBC}$ ) with platelets), as well as banking. A standard 2-h OGTT is performed after an 8to $12-\mathrm{h}$ fast using $75 \mathrm{~g}$ of glucose. Blood is drawn before and at 30, 60, 90, and 120 min after ingestion of the glucose load. The Matsuda index, which correlates well with the euglycemic clamp, is used to calculate insulin sensitivity [32]. Subjects are required to be fasting during each of these research visits.

\section{Exploratory outcomes}

The following exploratory outcomes are included:

1. Macrophage profiling

a) Macrophage abundance

b) Macrophage polarization state

2. Satellite cells
a) Abundance
b) Activation state
c) Fusion

3. Assessment of inflammation

a) Cytokine gene expression

b) Signal transduction

Macrophage profiling Resident muscle macrophage number and polarization state at baseline (first biopsy), after metformin or placebo only (second biopsy), and after 14 weeks of PRT (third biopsy) are determined with IHC. Subjects are asked to continue their normal activities of daily living but to refrain from any unaccustomed activity or exercise, including resistance training, through the first 2 weeks of the protocol to prevent any exercise-induced muscle inflammation. Pan monocyte/ macrophage antibody co-stains of CD11b and CD68, together with 4',6-diamidino-2-phenylindole (DAPI) staining, are used to quantify total macrophages in $7-\mu \mathrm{m}$ cryosections. The relative frequency of CD11b+/CD206-/ CD163- M1 pro-inflammatory macrophages to CD11b +/CD206+ M2 alternatively activated macrophages is also quantified. CD163 is used for the confirmation of CD206, 
M2 macrophages. The relative frequency of macrophage subtypes is then expressed per fiber area/total fibers.

Satellite cell analyses Muscle stem cell (satellite cells) abundance per fiber is quantified by IHC with the Pax7 monoclonal antibody. MyoD is expressed specifically in activated satellite cells, and IHC analysis of MyoD is used to identify activated satellite cells. These analyses are combined with counting total myofiber nuclei (DAPI-stained nuclei residing within the dystrophinlabeled sarcolemma) to monitor myonuclear accretion from satellite cell fusion that accompanies hypertrophic growth of myofibers in humans, including older adults.

Assessment of inflammation We are quantifying inflammatory gene expression in all subjects using the Nanostring nCounter analysis system. Approximately 100 genes are analyzed based on previous work of the genes that were most differentially expressed between the exercise responders and non-responders. Signaling pathways such as the AMPK pathway are measured by western blot using phospho-specific antibodies to Thr172 on AMPK. The mTORC pathway, antagonized by AMPK, is also analyzed, as it plays an important role in regulating protein synthesis in muscle. Other signaling pathways, such as p38 and PKC, are assessed in relation to growth and macrophage profile. Down-regulation of inflammatory signaling proteins, in particular, NFKB and STAT, will also be quantified.

\section{Statistical plan}

We intend to recruit 120 participants to account for a $20 \%$ attrition rate (84 UAB and $36 \mathrm{UK}$ ) to achieve a final sample size of 100 (50 metformin, 50 placebo). This allows sufficient power for "as observed" comparisons. Endpoints (myofiber CSA, thigh muscle CSA, strength, power, and macrophage abundance) and changes with training will be treated as continuous variables, summarized with descriptive statistics. SAS v9.2 or higher is being used for all analyses, and a significance level of 0.05 is used for all statistical tests. In the case that endpoints are found to be non-normal, appropriate transformations are employed and non-parametric tests used. The primary analysis and representative measure used to calculate power is the comparison of change in type II myofiber CSA for those randomized to either PRT with placebo or metformin. Type II myofiber CSA changes due to PRT with placebo or metformin will also be compared across sex. A two-sample $t$ test will have at least $80 \%$ power to detect an effect size of 0.6 when the sample size is 50 per group $(n=100)$, assuming a two-sided significance level of 0.05 . Based upon our prior resistance training trials in older adults, we expect 14 weeks of PRT alone to be associated with an approximately
20\% increase in type II myofiber CSA in both men and women. We predict that adjuvant metformin will yield an additional $25 \%$ increase in myofiber CSA (above the $20 \%$ due to PRT alone). Prior work (25 men and 25 women, age 65-80 years) showed pre-treatment type II CSA means (SD) of $4095(1213) \mu^{2}$ in men and 2458 (690) $\mu^{2}$ in women. With a $20 \%$ increase in PRT alone and an additional $25 \%$ increase with metformin, the mean difference between treatment groups would be at least $730 \mu \mathrm{m}^{2}$, assuming a common SD of 950 . The effect size is expected to be at least 0.76 , which is larger than the detectable effect size for our planned final sample of 100 .

\section{Statistics specific to primary outcomes}

The primary endpoint is the change in type II myofiber size after training, with the secondary outcome being change in muscle size. The primary analysis design is the intention-to-treat (ITT) comparison of change in myofiber/muscle size for those randomized to either placebo or metformin. It is expected that the randomization will lessen the need for covariate-adjusted analyses; however, in the event that adjusted analyses are necessary, a secondary comparison of the change in endpoints for the two groups will be made using analysis of covariance (ANCOVA). Potential confounders include baseline values, BMI, age, race, ethnicity, gender, changes in insulin sensitivity, and pill count. In addition to changes in muscle size, changes in strength are also of interest. Strength is measured at weeks 2, 4, 9, and 16 (training begins after 2 weeks of metformin or placebo). The primary analysis for strength is conducted using the same strategy as for muscle size, using a change score from week 4 (true strength baseline) to 16 . However, since additional time points are collected that investigate changes in strength over time between groups, the interaction of group and time can also be analyzed using covariance pattern models (or linear mixed models), where the correlation between observations measured over time can be handled more flexibly (i.e., unstructured and autoregressive variance-covariance matrices).

The ITT analysis is performed using all randomized participants regardless of loss to follow-up, where data for those lost is imputed using last observation carried forward (LOCF). In this case, LOCF should provide a conservative estimate of the effect; however, multiple imputation will also be used to impute missing values and to assess the sensitivity of the results based on LOCF. It is expected that subjects may not be compliant to the prescribed dosing, which is measured by pill count at specified visits. Non-compliant subjects are included in the ITT analysis; however, a modified ITT may also be conducted utilizing an ITT randomization principle but limited to those who achieved at least $1000 \mathrm{mg}$ per day. 
Thus, analyses are conducted comparing groups without imputed data ("as observed"), which will be impacted by attrition as well as ITT analysis using the randomized sample in which all participants are used, potentially allowing us to detect a smaller effect size (approximately 0.5 if $n=120$ ) with $80 \%$ power.

\section{Discussion}

As metformin is increasingly being used to treat medical conditions other than type 2 diabetes, identifying novel mechanisms of action of metformin is timely. While the mechanisms of action of metformin are not fully understood, much of the previous research has shown that metformin activates the enzyme AMPK, shown to be influential in glycemic control, energy balance, and metabolism in multiple peripheral tissues [33]. More recent animal research has shown metformin's profound effects on skeletal muscle through activation of AMPK. Improvements in structural integrity, oxidative metabolism, resiliency to muscle damage, and macrophage polarization have all been reported [34-36]. However, mouse models have shown that metformin can maintain its hypoglycemic effect in the absence of AMPK in the liver, indicating that metformin may act in an AMPK-independent manner [37]. Furthermore, metformin was shown to enhance mitochondrial respiration in skeletal muscle of AMPKdeficient mice after just 2 weeks of treatment [38]. Based on the wealth of research on the beneficial skeletal muscle effects and modulation of a variety of other conditions such as cardiovascular disease, cancer risk, and longevity, metformin has been proposed as a potential anti-aging drug [19, 39-41].

AMPK signaling also responds to exercise in skeletal muscle, but in an age- and sex-dependent manner. It has been demonstrated that AMPK activation is significantly increased in men but not in women following a bout of continuous submaximal aerobic exercise [42] and that aged animals show a reduced AMPK response to exercise [43]. Thus, results from this study will determine if metformin can augment the benefits of exercise in the elderly in an AMPK-dependent or AMPK-independent manner, and if this differs by sex. Results will also provide information on metformin tolerance and the frequency of GI side effects in both male and female healthy elderly individuals.

This will be the first metformin repurposing trial to test the potential synergistic impact of combined exercise-drug therapy on muscle mass and function, and it will be conducted in an aging cohort in need of a treatment that maximizes muscle regrowth and strength gain. This study has the potential to advance our understanding of the mechanisms involved in muscle adaption and to predict those individuals who may have trouble responding to an exercise training program. Age-related muscle inflammation susceptibility is a novel concept [6], which the study proposes as a central mechanism underlying the blunted responsiveness of many older adults to resistance training by promoting a catabolic environment. Our combined intervention will determine if this combined exercise-drug therapy will overcome the variable and non-responsive phenotype seen in aging. By imposing a two-site collaboration, the study becomes more generalizable and contains sufficient power not normally found in smaller resistance training trials.

In conclusion, there are several innovative features of the proposed experiments that are expected to significantly advance the field and improve muscle regrowth and mobility, with the overall goal of reducing risk of disability among older adults. The proposed work is expected to have a powerful impact, as we will be the first to determine whether metformin, in combination with resistance exercise designed to elicit muscle hypertrophy, will augment progressive resistance training-induced muscle gains in older adults and successfully restore function, health, and quality of life.

\section{Trial status}

The study has been active and open for enrollment since November 2014 with an anticipated completion date of December 2017.

\section{Additional file}

Additional file 1: SPIRIT checklist. (DOC $121 \mathrm{~kb}$ )

\section{Abbreviations}

ACSM: American College of Sports Medicine; AE: Adverse event; AMPK: 5' adenosine monophosphate-activated protein kinase; ATF-3: activating transcription factor 3; BMC: Bone mineral content; BMD: Bone mineral density; CBC: Complete blood cell count; CCTS: Center for Clinical and Translational Science; CSA: Crosssectional area; CT: Computed tomography; DAPI: 4',6-diamidino-2-phenylindole; DSMB: Data Safety and Monitoring Board; DXA: Dual energy x-ray absorptiometry; FFM: Free fat mass; FM: Fat mass; HIPAA: Health Insurance Portability and Accountability Act; HOMA: Homeostatic Model Assessment; HU: Hounsfield unit; IGF-1: Insulin growth factor 1; IHC: Immunohistochemistry; IL-10: Interleukin-10; IL-1ß: Interleukin-1 beta; IL-4: Interleukin-4; IL-6: Interleukin-6; IRB: Institutional review board; IT: Intent to treat; LOCF: Last observation carried forward:

LPS: Lipopolysaccharide; MFL: Mineral-free lean; mTORC: Mechanistic (formerly mammalian) target of rapamycin complex; MVIC: Maximum voluntary isometric contraction; MyHC: Myosin heavy chain; OGTT: Oral glucose tolerance test; PASE: Physical Activity Survey for the Elderly; PROMIS: Patient-Reported Outcomes Measurement Information System; PRT: Progressive resistance training; RM: Repetition maximum; ROI: Region of interest; SF-36: Short Form 36; SPPB: Short Physical Performance Battery; TNF-a: Tumor necrosis factor alpha; TSH: Thyroid-stimulating hormone; UAB: University of Alabama at Birmingham; UK: University of Kentucky; VA: Veterans Affairs

\section{Acknowledgements}

We would like to thank our participants for their time commitment, effort, and dedication.

\section{Funding}

The study was funded by the National Institutes of Health - National Institute on Aging grant AG046920 and supported by the NIH Clinical and Translational 
Science Awards (CTSA) (UL1TR001998) at the University of Kentucky and the NIH CTSA (UL1TR000165) at the University of Alabama at Birmingham. The NIH had no role in the design of the study, collection, analysis, or interpretation of data, or in writing the manuscript.

\section{Availability of data and materials}

The supporting data used for preliminary analyses are available from the corresponding author on reasonable request.

\section{Authors' contributions}

The protocol authors, DEL, BDP, and CAP, wrote the manuscript; DEL, HMB, $J \mathrm{LM}, \mathrm{SCT}, \mathrm{GM}, \mathrm{PAK}, \mathrm{MMB}$, and CAP all have contributed to the design, planning, and/or implementation of the protocol. All authors read and approved the final manuscript.

\section{Competing interests}

The authors declare that they have no competing interests. The manufacturers' of the products involved in the study did not contribute to the design, implementation, or support of the study or study investigators in any way.

\section{Consent for publication}

Not applicable.

\section{Ethics approval and consent to participate}

This study was approved by the University of Kentucky institutional review board (IRB 14-0330) and the University of Alabama at Birmingham institutional review board (IRB F140722001) prior to any subjects enrolling. Data and safety monitoring is provided by the UK CCTS DSMB on a quarterly basis. All participation is on a voluntary basis, and each participant is required to sign an approved IRB consent form and HIPAA authorization prior to their participation in the study protocol.

\section{Publisher's Note}

Springer Nature remains neutral with regard to jurisdictional claims in published maps and institutional affiliations.

\section{Author details}

${ }^{1}$ College of Health Sciences and Center for Muscle Biology, University of Kentucky, Lexington, KY, USA. ${ }^{2}$ Center for Exercise Medicine and Department of Cell, Developmental, and Integrative Biology, University of Alabama at Birmingham, Birmingham, AL, USA. ${ }^{3}$ Department of Biostatistics, College of Public Health, University of Kentucky, Lexington, KY, USA. ${ }^{4}$ Department of Internal Medicine, Division of Endocrinology, and Barnstable Brown Diabetes and Obesity Center, University of Kentucky, Lexington, KY, USA.

\section{Received: 9 November 2016 Accepted: 6 April 2017}

\section{Published online: 26 April 2017}

\section{References}

1. Mitchell WK, Williams J, Atherton P, Larvin M, Lund J, Narici M. Sarcopenia, dynapenia, and the impact of advancing age on human skeletal muscle size and strength; a quantitative review. Front Physiol. 2012;3:260.

2. de Labra C, Guimaraes-Pinheiro C, Maseda A, Lorenzo T, Millán-Calenti JC. Effects of physical exercise interventions in frail older adults: a systematic review of randomized controlled trials. BMC Geriatr. 2015;15:154

3. Lustosa LP, Silva JP, Coelho FM, Pereira DS, Parentoni AN, Pereira LSM. Impact of resistance exercise program on functional capacity and muscular strength of knee extensor in pre-frail community-dwelling older women: a randomized crossover trial. / Efeito de um programa de resistência muscular na capacidade funcional e na força muscular dos extensores do joelho em idosas pré-frágeis da comunidade: ensaio clínico aleatorizado do tipo crossover. Braz J Phys Ther / Rev Bras Fisioter. 2011;15:318-24.

4. Bamman MM, Petrella JK, Jeong-su K, Mayhew DL, Cross JM. Cluster analysis tests the importance of myogenic gene expression during myofiber hypertrophy in humans. J Appl Physiol. 2007;102:2232-9.

5. Dennis RA, Przybyla B, Gurley C, et al. Aging alters gene expression of growth and remodeling factors in human skeletal muscle both at rest and in response to acute resistance exercise. Physiol Genomics. 2008;32:393-400.

6. Merritt EK, Stec MJ, Thalacker-Mercer A, et al. Heightened muscle inflammation susceptibility may impair regenerative capacity in aging humans. J Appl Physiol. 2013;115:937-48
7. Petrella JK, Jeong-su K, Mayhew DL, Cross JM, Marcas MB. Potent myofiber hypertrophy during resistance training in humans is associated with satellite cell-mediated myonuclear addition: a cluster analysis. J Appl Physiol. 2008; 104:1736-42.

8. Dennis RA, Zhu H, Kortebein PM, et al. Muscle expression of genes associated with inflammation, growth, and remodeling is strongly correlated in older adults with resistance training outcomes. Physiol Genomics. 2009:38:169-75.

9. Fisher G, Bickel CS, Hunter GR. Elevated Circulating TNF-a in Fat-Free Mass Non-Responders Compared to Responders Following Exercise Training in Older Women. Biology. 2014;3:551-9.

10. Przybyla B, Gurley C, Harvey JF, et al. Aging alters macrophage properties in human skeletal muscle both at rest and in response to acute resistance exercise. Exp Gerontol. 2006;41:320-7.

11. Arnold L, Henry A, Poron F, et al. Inflammatory monocytes recruited after skeletal muscle injury switch into antiinflammatory macrophages to support myogenesis. J Exp Med. 2007;204:1057-69.

12. Robertson TA, Maley MA, Grounds MD, Papadimitriou JM. The role of macrophages in skeletal muscle regeneration with particular reference to chemotaxis. Exp Cell Res. 1993;207:321-31.

13. Tidball JG, Wehling-Henricks M. Macrophages promote muscle membrane repair and muscle fibre growth and regeneration during modified muscle loading in mice in vivo. J Physiol. 2007:578:327-36.

14. Paylor B, Natarajan A, Zhang RH, Rossi F. Nonmyogenic cells in skeletal muscle regeneration. Curr Top Dev Biol. 2011;96:139-65.

15. Dumont N, Frenette J. Macrophages protect against muscle atrophy and promote muscle recovery in vivo and in vitro: a mechanism partly dependent on the insulin-like growth factor-1 signaling molecule. Am J Pathol. 2010;176: 2228-35.

16. Cantini M, Giurisato E, Radu C, et al. Macrophage-secreted myogenic factors: a promising tool for greatly enhancing the proliferative capacity of myoblasts in vitro and in vivo. Neurol Sci. 2002;23:189-94.

17. Chazaud B, Sonnet C, Lafuste $P$, et al. Satellite cells attract monocytes and use macrophages as a support to escape apoptosis and enhance muscle growth. J Cell Biol. 2003;163:1133-43.

18 Check HE. Anti-ageing pill pushed as bona fide drug. Nature. 2015:522:265-6.

19 Bannister CA, Holden SE, Jenkins-Jones S, et al. Can people with type 2 diabetes live longer than those without? A comparison of mortality in people initiated with metformin or sulphonylurea monotherapy and matched, non-diabetic controls. Diabetes Obes Metab. 2014;16:1165-73.

20 Kim YD, Park K-G, Lee Y-S, et al. Metformin inhibits hepatic gluconeogenesis through AMP-activated protein kinase-dependent regulation of the orphan nuclear receptor SHP. Diabetes. 2008;57:306-14.

21 Kirpichnikov D, McFarlane SI, Sowers JR. Metformin: an update. Ann Intern Med. 2002;137:25-33.

22 Zhou G, Myers R, Li Y, et al. Role of AMP-activated protein kinase in mechanism of metformin action. J Clin Invest. 2001;108:1167-74.

23 Mounier R, Théret M, Lantier L, Foretz M, Viollet B. Expanding roles for AMPK in skeletal muscle plasticity. Trends Endocrinol Metab. 2015:26:275-86.

24 Mounier $R$, Théret $M$, Arnold $L$, et al. AMPKa1 regulates macrophage skewing at the time of resolution of inflammation during skeletal muscle regeneration. Cell Metab. 2013;18:251-64.

$25 \mathrm{Kim}$ J, Kwak HJ, Cha J-Y, et al. Metformin suppresses lipopolysaccharide (LPS)-induced inflammatory response in murine macrophages via activating transcription factor-3 (ATF-3) induction. J Biol Chem. 2014:289:23246-55.

26 Guralnik JM, Simonsick EM, Ferrucci L, et al. A short physical performance battery assessing lower extremity function: association with self-reported disability and prediction of mortality and nursing home admission. J Gerontol. 1994:49:M85-94

27 Blanton CA, Moshfegh AJ, Baer DJ, Kretsch MJ. The USDA Automated Multiple-Pass Method accurately estimates group total energy and nutrient intake. J Nutr. 2006;136:2594-9.

28 Bickel CS, Cross JM, Bamman MM. Exercise dosing to retain resistance training adaptations in young and older adults. Med Sci Sports Exerc. 2011; 43:1177-87.

29 Bamman MM, Clarke MSF, Feeback DL, et al. Impact of resistance exercise during bed rest on skeletal muscle sarcopenia and myosin isoform distribution. J Appl Physiol. 1998:84:157-63.

30 Petrella JK, Jeong-su K, Tuggle SC, Hall SR, Bamman MM. Age differences in knee extension power, contractile velocity, and fatigability. J Appl Physiol. 2005;98:211-20 
31 Srikuea R, Symons TB, Long DE, et al. Association of fibromyalgia with altered skeletal muscle characteristics which may contribute to postexertional fatigue in postmenopausal women. Arthritis Rheum. 2013;65:519-28.

32 Matsuda M, Liu Y, Mahankali S, et al. Altered hypothalamic function in response to glucose ingestion in obese humans. Diabetes. 1999;48:1801-6.

33 Towler MC, Hardie DG. AMP-activated protein kinase in metabolic control and insulin signaling. Circ Res. 2007;100:328-41.

34 Jahnke VE, Van Der Meulen JH, Johnston HK, et al. Metabolic remodeling agents show beneficial effects in the dystrophin-deficient mdx mouse model. Skelet Muscle. 2012;2:16-26.

35 Langone F, Cannata S, Fuoco C, et al. Metformin protects skeletal muscle from cardiotoxin induced degeneration. PLoS One. 2014;9:1-19.

36 Lantier L, Fentz J, Mounier R, et al. AMPK controls exercise endurance, mitochondrial oxidative capacity, and skeletal muscle integrity. FASEB J. 2014:28:3211-24.

37 Foretz M, Hébrard S, Leclerc J, et al. Metformin inhibits hepatic gluconeogenesis in mice independently of the LKB1/AMPK pathway via a decrease in hepatic energy state. J Clin Invest. 2010;120:2355-69.

38 Kristensen JM, Larsen S, Helge JW, Dela F, Wojtaszewski JFP. Two weeks of metformin treatment enhances mitochondrial respiration in skeletal muscle of AMPK kinase dead but not wild type mice. PLoS One. 2013;8:e53533.

39 Anisimov VN. Metformin: do we finally have an anti-aging drug? Cell Cycle (Georgetown, Tex). 2013;12:3483-9.

40 Evans JMM, Donnelly LA, Emslie-Smith AM, Alessi DR, Morris AD. Metformin and reduced risk of cancer in diabetic patients. BMJ (Clinical Research Ed). 2005;330:1304-5.

41 Morgan CL, Jenkins-Jones S, Holden SE, Currie CJ, Mukherjee J. Association between first-line monotherapy with sulphonylurea versus metformin and risk of all-cause mortality and cardiovascular events: a retrospective, observational study. Diabetes Obes Metab. 2014;16:957-62.

42 Roepstorff C, Thiele M, Hillig T, et al. Higher skeletal muscle a2AMPK activation and lower energy charge and fat oxidation in men than in women during submaximal exercise. J Physiol. 2006;574:125-38.

43 Reznick RM, Zong H, Li J, et al. Aging-associated reductions in AMP-activated protein kinase activity and mitochondrial biogenesis. Cell Metab. 2007;5:151-6.

\section{Submit your next manuscript to BioMed Central and we will help you at every step:}

- We accept pre-submission inquiries

- Our selector tool helps you to find the most relevant journal

- We provide round the clock customer support

- Convenient online submission

- Thorough peer review

- Inclusion in PubMed and all major indexing services

- Maximum visibility for your research

Submit your manuscript at www.biomedcentral.com/submit

) Biomed Central 